\title{
An ISO investigation of the MWC 297 circumstellar region ${ }^{\star}$
}

\author{
M. Benedettini ${ }^{1}$, S. Pezzuto ${ }^{1}$, T. Giannini ${ }^{2}$, D. Lorenzetti ${ }^{2}$, and B. Nisini ${ }^{2}$ \\ 1 CNR-Istituto di Fisica dello Spazio Interplanetario, Area di Ricerca di Tor Vergata, \\ via del Fosso del Cavaliere 100, 00133, Roma, Italy \\ 2 Osservatorio Astronomico di Roma, via Frascati 33, 00040, Monte Porzio, Italy
}

Received 16 July 2001 / Accepted 20 September 2001

\begin{abstract}
The ISO-SWS full grating spectrum $(2.3-45 \mu \mathrm{m})$ of the Herbig Be star MWC 297 is presented. The spectrum is dominated by a strong continuum with superimposed emission lines and features both in absorption and in emission. In particular, we detect in emission $23 \mathrm{HI}$ recombination lines of the Brackett, Pfund and Humphreys series and four PAH features, while in absorption two broad silicate bands at 9.7 and $16.4 \mu \mathrm{m}$, solid $\mathrm{CO}_{2}$ at $4.27 \mu \mathrm{m}$ and solid $\mathrm{H}_{2} \mathrm{O}$ at $2.96 \mu \mathrm{m}$ have been observed. The ISO-SWS spectrum has been combined with ISO-LWS data and ground based photometry to derive the spectral energy distribution (SED) from optical to radio wavelengths. The observed SED has been fitted with a model that assumes a spherical dusty envelope parametrized by density and temperature power laws, deriving suitable values for the spectral type (B2), the visual extinction $(7.5 \mathrm{mag})$ and the distance $(280 \mathrm{pc})$. Consistent determination of the extinction and estimates of both the source mass loss rate $\left(9 \times 10^{-7} M_{\odot} \mathrm{yr}^{-1}\right)$ and the size of the emitting ionized region (30 stellar radii) have been derived by the analysis of the $\mathrm{H}$ I recombination lines of the Brackett, Pfund and Humphreys series observed by ISO-SWS together with Paschen and Brackett lines observed from the ground. Some peculiarities have been observed in the ISO-SWS spectrum of this source: $i$ ) the ratio between the column density of the solid $\mathrm{CO}_{2}$ and $\mathrm{H}_{2} \mathrm{O}(2.0 \pm 1.5)$ higher than the values usually observed and $i$ ) the presence of a silicate broad absorption band at $16.4 \mu \mathrm{m}$ stronger than the $9.7 \mu \mathrm{m}$ absorption. The observed silicate absorption features are probably due to the extended dusty cloud in which the star is embedded and their relative strengths could be evidence that they are composed by processed grains.
\end{abstract}

Key words. stars: circumstellar matter - stars: individual: MWC 297 - infrared: ISM: lines and bands

\section{Introduction}

MWC 297 is a highly reddened object whose optical spectrum is characterized by the presence of hundreds of atomic emission lines (Andrillat \& Jaschek 1998). The $\mathrm{H} \alpha$ line is extremely strong and broadened (Drew et al. 1997), indicating the presence of strong winds in the stellar environment. This source has originally been classified as a pre-Main Sequence object of intermediate mass and was inserted in the original list of Herbig Ae/Be (HAEBE) stars (Herbig 1960); however its evolutionary state, as well as other important physical parameters, are not yet accurately known. Bergen et al. (1988) classified MWC 297 as a B0 star and Cantó et al. (1984) derived a distance of 450 pc. More recently Drew et al. (1997), from the analysis of optical spectra, revised this view attributing

Send offprint requests to: M. Benedettini,

e-mail: milena@ifsi.rm.cnr.it

* Based on observations with ISO, an ESA project with instruments funded by ESA Member States and with the participation of ISAS and NASA. to the object a spectral type B1.5, a distance of $250 \mathrm{pc}$ and a later evolutionary state. The $5 \mathrm{GHz}$ radio map by Drew et al. (1997) shows that the source is elongated in the north-south direction $\left(0.31 \times 0.20 \operatorname{arcsec}^{2}\right)$ suggesting the presence of an equatorial disc. The absence of polarization changes across the $\mathrm{H} \alpha$ line (Oudmaijer \& Drew 1999) would imply however that the ionized envelope is spherically symmetric. KAO images by Di Francesco et al. (1998) show the presence of a quite extended FIR circumstellar region, whose size is $\sim 60 \times 50 \operatorname{arcsec}^{2}$. The dusty envelope has been resolved also at NIR wavelengths by interferometric observations in the $H$ and $K^{\prime}$ bands (MillanGabet et al. 2001). The $1.3 \mathrm{~mm}$ map by Henning et al. (1998) reveals a central core at the same position of the optical star, surrounded by a more extended envelope.

We observed MWC 297 with the two spectrometers onboard the Infrared Space Observatory (ISO, Kessler et al. 1996). The Long Wavelength Spectrometer (LWS, Clegg et al. 1996) spectrum (43-197 $\mu \mathrm{m})$ presents the two [O I] lines at $63 \mu \mathrm{m}$ and $145 \mu \mathrm{m}$ and the [C II] line at $158 \mu \mathrm{m}$ (Lorenzetti et al. 1999). The ratio between the 


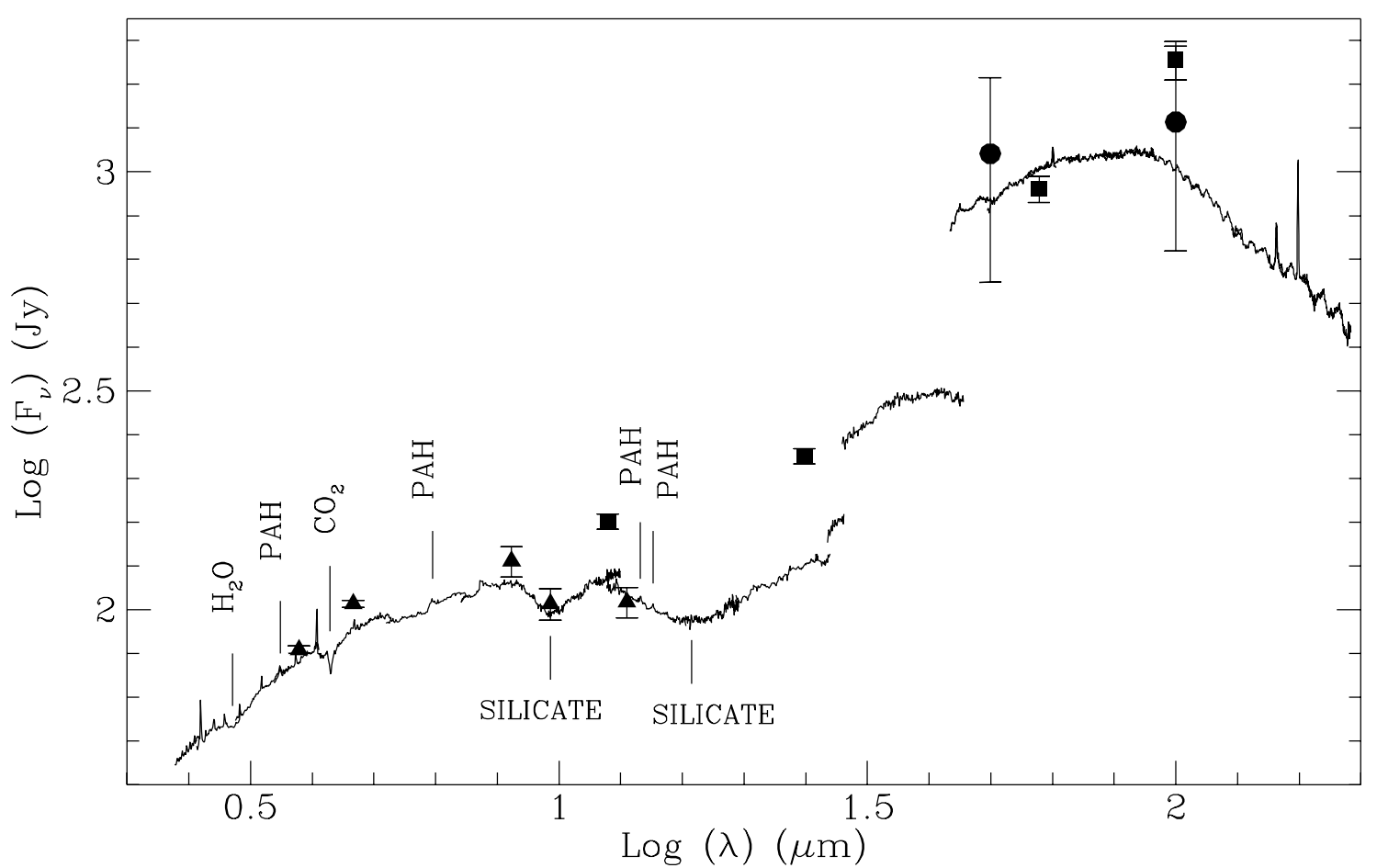

Fig. 1. ISO-SWS $(2.3-45 \mu \mathrm{m})$ and ISO-LWS $(43-197 \mu \mathrm{m})$ spectra of MWC 297, where the single spectra of the 10 LWS detectors have been stitched together. Triangles are ground based photometry (Berrilli et al. 1992), squares are IRAS Point Source Catalog photometry and filled circles are KAO data (Di Francesco et al. 1998).

two [O I] lines ([O I] $63 \mu \mathrm{m} /[\mathrm{O} \mathrm{I}] 145 \mu \mathrm{m}=2.0 \pm 0.2)$ is extremely low with respect to the predictions of both PhotoDissociation Regions (Kaufman et al. 1999) and shocks (Hollenbach \& McKee 1989). Lorenzetti et al. (1999) suggest that the observed ratio could be due to a strong absorption of the [O I] $63 \mu \mathrm{m}$ line by cold $(\sim 100 \mathrm{~K})$ atomic oxygen present along the line of sight.

In this paper we analyze the $2.3-45 \mu \mathrm{m}$ spectrum of MWC 297, provided by the Short Wavelength Spectrometer (SWS, de Graauw et al. 1996) in order to derive information about the distribution and the physical parameters of the circumstellar envelope and to investigate the properties of the ionized wind which characterizes the early stage of the stellar evolution. In Sect. 2 the observations and the reduction method are described and in Sect. 3 the results are presented. The results are analyzed and discussed in Sect. 4 and the conclusions are given in Sect. 5 .

\section{Observations and data reduction}

MWC 297 (RA(2000): 18h27m39.5s; DEC(2000): $\left.-03^{\circ} 49^{\prime} 52.1^{\prime \prime}\right)$ was observed with SWS and LWS onboard the ISO satellite on the 23rd October 1997. The SWS observations were carried out with the AOT01 full grating scan mode at speed 2, corresponding to a resolution from 250 to 600 ; the LWS spectrum was obtained with the AOT01 full grating scan mode with a resolution $\sim 200$. Raw satellite data have been reduced by using the ISO-SWS Interactive Analysis procedures (OSIA1.0) and the ISO Spectral Analysis Package (ISAP1.6).
The wavelength and flux calibrations were based on the calibration files available in version 7 of the Off-Line Processing pipeline for the SWS data and version 9 for the LWS data. The corresponding wavelength calibration accuracy for the SWS spectrum is better than $1 / 5$ of the resolution element (Valentijn et al. 1996) and the absolute flux calibration accuracy ranges from $7 \%$ in the short wavelength $(<4.1 \mu \mathrm{m})$ part of the spectrum to $\sim 30 \%$ in the long wavelength $(>29 \mu \mathrm{m})$ part (Leech et al. 1997). Spurious signals due to the cosmic ray impacts were removed and the data of the 12 detectors were averaged together and rebinned at about twice the nominal instrumental resolution. As regards the LWS data, we concentrate our interest on the continuum spectrum because the emission lines have already been analyzed by Lorenzetti et al. (1999). The overall flux accuracy of the LWS data is $\sim 30 \%$; the final spectrum has been obtained averaging the scans of each detector after the de-glitching procedure.

\section{Results}

The ISO-SWS spectrum of MWC 297 (see Fig. 1) is dominated by a strong continuum with superimposed emission lines from the $\mathrm{H}$ I recombination series, $\mathrm{PAH}$ emission and absorption by solid $\mathrm{CO}_{2}, \mathrm{H}_{2} \mathrm{O}$ and silicates. We consider as a detection a feature or a line with signal to noise ratio greater than 3 ; for the lines a second requirement is that the $F W H M$ is comparable with the nominal instrumental resolution.

Figure 2 shows the continuum subtracted portion of the ISO-SWS spectrum in which the following 

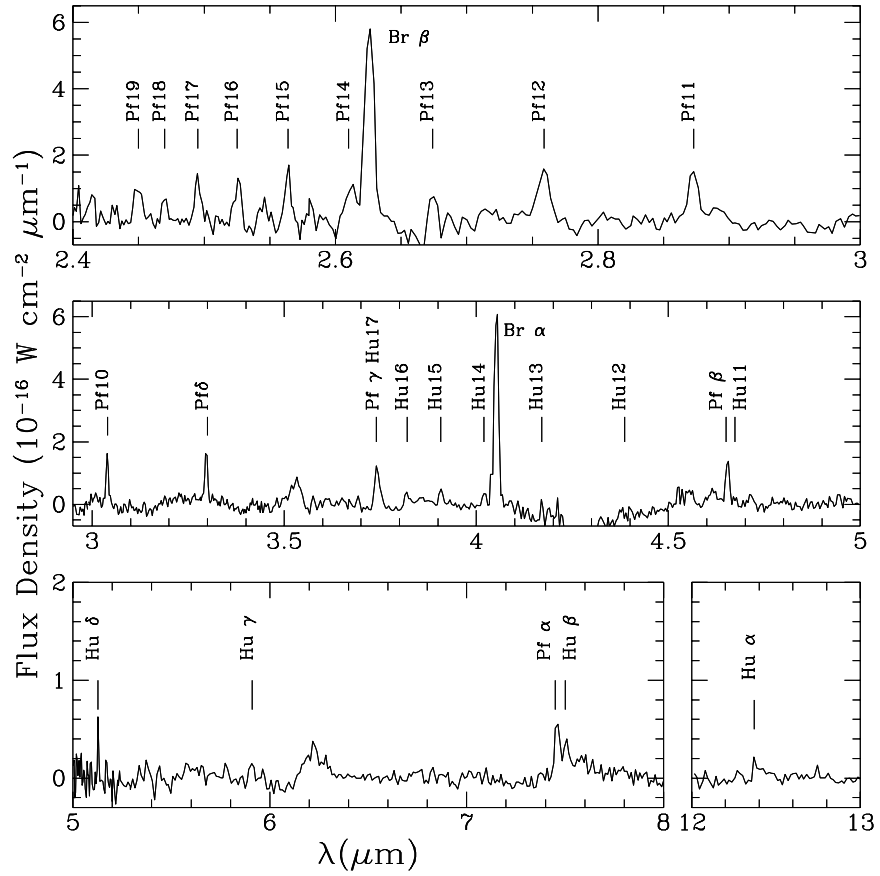

Fig. 2. Portions of the continuum subtracted spectrum of MWC 297 where the Hi recombination lines have been detected.

H I recombination lines have been detected: $\operatorname{Br} \alpha, \operatorname{Br} \beta$, Pfund lines from Pf $\alpha$ to Pf19 and Humphreys lines from $\mathrm{Hu} \alpha$ to $\mathrm{Hu} \delta$ plus Hu14, 15 and 16. These lines are listed in Table 1 (Col. 1) together with the fitted central wavelength (Col. 2) and the integrated flux (computed by a Gaussian fitting) with the associated $1 \sigma$ statistical error (Col. 3), which refers to the rms noise of the local baseline; for the Hu11, 12 and 13 lines the $3 \sigma$ upper limit is given. The $\mathrm{Pf} \gamma$ is blended with the Hu17, whose flux is expected to be smaller than the Hu16 flux $\left(0.5 \times 10^{-18} \mathrm{~W} \mathrm{~cm}^{-2}\right)$; thus we consider the calculated line flux $\left(2.1 \times 10^{-18} \mathrm{~W} \mathrm{~cm}^{-2}\right)$ as an upper limit for the $\operatorname{Pf} \gamma$ flux. All the lines are observed with the same beam aperture of $14^{\prime \prime} \times 20^{\prime \prime}$ with the exception of the $\mathrm{Hu} \alpha$ at $12.38 \mu \mathrm{m}$ for which the aperture is slightly larger $\left(14^{\prime \prime} \times 27^{\prime \prime}\right)$; nevertheless, in the analysis we do not apply any beam correction to the Hu $\alpha$ line because it is expected that the region responsible for the $\mathrm{H}$ I recombination lines is much smaller than the beam aperture.

The comparison of the ISO-SWS Br $\alpha$ flux with groundbased observations by Nisini et al. (1995) $\left(F_{\mathrm{Br} \alpha}=(6.14 \pm\right.$ $\left.0.76) \times 10^{-18} \mathrm{~W} \mathrm{~cm}^{-2}\right)$ and by Drew et al. (1997) $\left(F_{\mathrm{Br} \alpha}=\right.$ $1.1 \times 10^{-17} \mathrm{~W} \mathrm{~cm}^{-2}$ ), indicates a variability of this line, also observed in the $\mathrm{H} \alpha$ line (Drew et al. 1997).

Other features detected in the ISO-SWS spectrum are: ice absorption at $2.96 \mu \mathrm{m}$ and $4.27 \mu \mathrm{m}$ associated with $\mathrm{O}-\mathrm{H}$ stretching mode of solid $\mathrm{H}_{2} \mathrm{O}$ and $\mathrm{C}=\mathrm{O}$ asymmetric stretching vibration of solid $\mathrm{CO}_{2}$, respectively and two broad absorption silicate bands between $8-11 \mu \mathrm{m}$ and $12-23 \mu \mathrm{m}$; the first, peaking at $9.7 \mu \mathrm{m}$, is associated to the $\mathrm{Si}-\mathrm{O}$ stretching vibrations in amorphous silicates, the second to the $\mathrm{O}-\mathrm{Si}-\mathrm{O}$ bending mode and peaks at $16.4 \mu \mathrm{m}$.
Table 1. Fluxes of the H I recombination lines observed in the ISO-SWS spectrum. Upper limits are $3 \sigma$.

\begin{tabular}{lcc}
\hline line id. & $\begin{array}{c}\lambda_{\text {obs }} \\
(\mu \mathrm{m})\end{array}$ & $\begin{array}{c}F \pm \Delta F \\
\left(10^{-18} \mathrm{~W} \mathrm{~cm}^{-2}\right)\end{array}$ \\
\hline \hline Br $\alpha$ & 4.052 & $7.5 \pm 0.2$ \\
Br $\beta$ & 2.626 & $5.5 \pm 0.3$ \\
\hline Pf $\alpha$ & 7.460 & $1.3 \pm 0.2$ \\
Pf $\beta$ & 4.654 & $1.6 \pm 0.1$ \\
Pf $\gamma$-Hu17 & 3.742 & $2.1 \pm 0.2$ \\
Pf $\delta$ & 3.297 & $1.42 \pm 0.09$ \\
Pf10 & 3.039 & $1.19 \pm 0.09$ \\
Pf11 & 2.873 & $1.2 \pm 0.1$ \\
Pf12 & 2.759 & $1.4 \pm 0.2$ \\
Pf13 & 2.674 & $0.9 \pm 0.2$ \\
Pf14 & 2.612 & $1.0 \pm 0.2$ \\
Pf15 & 2.564 & $0.8 \pm 0.1$ \\
Pf16 & 2.526 & $0.7 \pm 0.1$ \\
Pf17 & 2.495 & $0.7 \pm 0.1$ \\
Pf18 & 2.470 & $0.3 \pm 0.1$ \\
Pf19 & 2.450 & $0.5 \pm 0.1$ \\
\hline Hu $\alpha$ & 12.38 & $0.6 \pm 0.2$ \\
Hu $\beta$ & 7.703 & $0.9 \pm 0.2$ \\
Hu $\gamma$ & 5.908 & $0.5 \pm 0.2$ \\
Hu $\delta$ & 5.129 & $0.6 \pm 0.1$ \\
Hu11 & 4.673 & $<0.6$ \\
Hu12 & 4.376 & $<0.6$ \\
Hu13 & 4.170 & $<0.6$ \\
Hu14 & 4.021 & $0.49 \pm 0.07$ \\
Hu15 & 3.908 & $0.52 \pm 0.07$ \\
Hu16 & 3.819 & $0.5 \pm 0.1$ \\
\hline \hline
\end{tabular}

$\dagger$ Blended lines.

In addition, emission features from Polyciclic Aromatic Hydrocarbons (PAHs) at 3.53, 6.22, 13.56 and $14.21 \mu \mathrm{m}$ have been detected. In Table 2 the detected features are listed together with the parameters derived from the fit of their profile: the central wavelength (Col. 2), the integrated flux with its $1 \sigma$ statistical error (Col. 3) and the FWHM (Col. 4).

Looking at the ISO-SWS spectra of HAEBE stars (Meeus et al. 2001) it can be noticed that the NIR range is very similar for all the objects while in the MIR and FIR regions a large diversity in the spectral features and shape is revealed. In particular, in some of the sources of the Meeus et al. sample a blackbody continuum component, features emission of PAHs and silicates are not present. In MWC 297 we detect PAHs emission but the two silicate bands are in absorption; silicate absorption at $9.7 \mu \mathrm{m}$ has been detected also in the HAEBE star $\mathrm{LkH} \alpha 225$ (van den Ancker et al. 2000). 
Table 2. Features observed in the ISO-SWS spectrum.

\begin{tabular}{cccc}
\hline id. & $\begin{array}{c}\lambda_{\text {obs }} \\
(\mu \mathrm{m})\end{array}$ & $\begin{array}{c}F \pm \Delta F \\
\left(10^{-18} \mathrm{~W} \mathrm{~cm}{ }^{-2}\right)\end{array}$ & $\begin{array}{c}F W H M \\
(\mu \mathrm{m})\end{array}$ \\
\hline $\mathrm{H}_{2} \mathrm{O}^{\dagger}$ & 2.96 & $7 \pm 2$ & 0.1 \\
$\mathrm{PAH}^{\ddagger}$ & 3.53 & $2.3 \pm 0.4$ & 0.03 \\
$\mathrm{CO}_{2}^{\dagger}$ & 4.27 & $7.5 \pm 0.8$ & 0.05 \\
$\mathrm{PAH}^{\ddagger}$ & 6.22 & $3.6 \pm 0.5$ & 0.1 \\
silicate $^{\dagger}$ & $9.7^{*}$ & - & $\sim 1.4$ \\
$\mathrm{PAH}^{\ddagger}$ & 13.56 & $0.6 \pm 0.2$ & 0.08 \\
$\mathrm{PAH}^{\ddagger}$ & 14.21 & $0.6 \pm 0.2$ & 0.1 \\
silicate $^{\dagger}$ & $16.4^{*}$ & - & $\sim 5.5$ \\
\hline
\end{tabular}

$\dagger$ Absorption feature.

\# Emission feature.

${ }^{*}$ Wavelength of the absorption peak.

\section{Analysis and discussion}

\subsection{Continuum}

The ISO-SWS and ISO-LWS spectra of MWC 297 are shown in Fig. 1 together with ground based photometry by Berrilli et al. (1992) in the $L\left(\lambda_{\mathrm{eff}}=3.79 \mu \mathrm{m}\right), M\left(\lambda_{\mathrm{eff}}=\right.$ $4.64 \mu \mathrm{m}), N 1\left(\lambda_{\text {eff }}=8.38 \mu \mathrm{m}\right), N 2\left(\lambda_{\text {eff }}=9.69 \mu \mathrm{m}\right), N 3$ $\left(\lambda_{\text {eff }}=12.89 \mu \mathrm{m}\right)$ bands, the IRAS Point Source Catalog intensity at 12, 25, 60 and $100 \mu \mathrm{m}$ and the KAO data at 50 and $100 \mu \mathrm{m}$ (Di Francesco et al. 1998). The ground based photometry is in agreement with the ISO data, within the SWS flux calibration accuracy, with the exception of the $M$ band, but it can be due to the strong sky absorption variability in this band. At variance, the IRAS fluxes have significantly larger values with respect to the ISO ones, except for the $60 \mu \mathrm{m}$ flux. In the SWS range this difference can be accounted for by considering that the IRAS beam apertures are much larger than the ISO apertures $\left(14^{\prime \prime} \times 20^{\prime \prime}\right.$ for $2.3<\lambda<12 \mu \mathrm{m}, 14^{\prime \prime} \times 27^{\prime \prime}$ for $12<\lambda<27 \mu \mathrm{m}, 20^{\prime \prime} \times 27^{\prime \prime}$ for $27<\lambda<29 \mu \mathrm{m}, 20^{\prime \prime} \times 33^{\prime \prime}$ for $29<\lambda<45 \mu \mathrm{m}$ and $\sim 80^{\prime \prime}$ for $43<\lambda<197 \mu \mathrm{m}$ ) and that the emitting source is more extended than the ISOSWS apertures, as it can be inferred by considering that whenever the source is observed with a larger aperture, a rise in the flux level is revealed (e.g. the gap at $27 \mu \mathrm{m}$ between the SWS bands 2 and 3, the gap at $29 \mu \mathrm{m}$ between the SWS bands 3 and 4 and the gap at $45 \mu \mathrm{m}$ between SWS and LWS). KAO images by Di Francesco et al. (1998) indicates that the size $(F W H M)$ of the source at $50 \mu \mathrm{m}$ is $(57 \pm 13) \times(46 \pm 15) \operatorname{arcsec}^{2}$, with a total flux density of $1100 \pm 540 \mathrm{Jy}$ and $(61 \pm 11) \times(54 \pm 14) \operatorname{arcsec}^{2}$ at $100 \mu \mathrm{m}$ with a total flux density of $1300 \pm 640 \mathrm{Jy}$; these values are consistent with the ISO-LWS ones. In particular, the $100 \mu \mathrm{m}$ image reveals the presence of a diffuse background emission which can account for the higher value measured by IRAS. The consistency between the ISO-SWS and the ground based photometry testifies for a no variability of the continuum emission at near and mid infrared wavelengths, as also found in the visible range, with the exception of the $U$ band in which a variability is reported (Bergen et al. 1988).

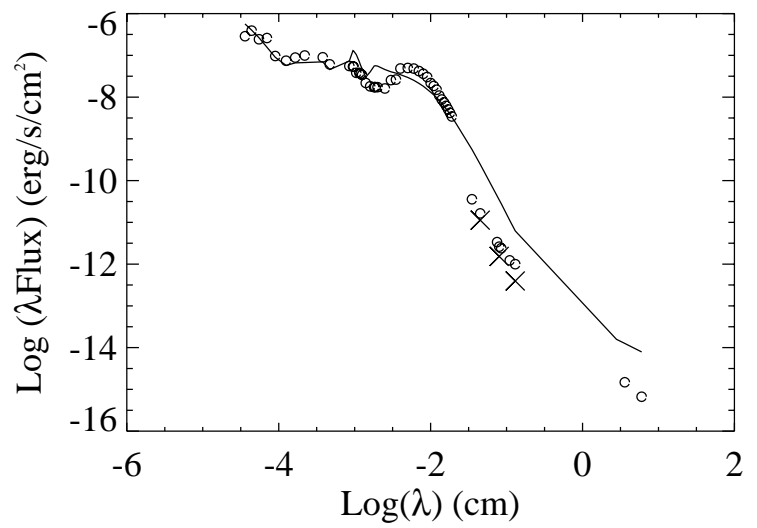

Fig. 3. SED of MWC 297: circles are ground base photometric measurements and ISO data (binned with steps of about $10 \mu \mathrm{m}$ ), solid line is the model and crosses are the model after taking into account the beam size (diffraction) at (sub)mm wavelengths.

The ISO data have been complemented with photometric measurements from optical to radio wavelengths to derive the spectral energy distribution (SED) of MWC 297. The observed SED has been compared with the SED's computed with a spherical dusty envelope model in order to probe if such a circumstellar matter distribution is compatible with the observations, deriving also suitable values for the parameters of the circumstellar matter as well as of the central source. The temperature and density distributions of the circumstellar envelope are parametrized by a radial power law with index $q$ and $p$, respectively. A detailed description of the model and references of the photometric data are given in Pezzuto et al. (1997).

By fitting the observed SED (Fig. 3) the following parameters have been derived: $q=0.5, p=1.1, \mathrm{SpT}=\mathrm{B} 2$, distance $D=280 \mathrm{pc}$ and visual extinction $A_{\mathrm{V}}=7.5 \mathrm{mag}$. The model has a circumstellar extinction, due to its own envelope, of $0.5 \mathrm{mag}$, while the interstellar extinction, found as part of the best fit search, is 7.0 mag. This means that to fit all the data from optical bands to radio wavelengths, a model with a small amount of circumstellar dust has been selected. As a consequence, the silicate bands of the model are in emission whereas in the spectrum absorption features have been observed and, in general, the agreement between the observational data and our model is quite poor in the region around $10 \mu \mathrm{m}$. It is worth noting that in a sample of ISO-SWS spectra of HAEBE stars (Meeus et al. 2001) the silicate features appear in emission for the more evolved and not embedded objects, while the silicate at $9.7 \mu \mathrm{m}$ has been detected in absorption in $\mathrm{LkH} \alpha 225$ (van den Ancker et al. 2000). As MWC 297, as well as $\mathrm{LkH} \alpha 225$, is surrounded by an extended dusty cloud, the silicate absorption can be ascribed to this extended component rather than to the star envelope. If we assume that the extended component is distinct from MWC 297, in the sense that its temperature and density are not directly related to the physical properties of the 
circumstellar dust around MWC 297, it is not surprising that our model fails to reproduce the observed silicate absorption bands. In any case, the addition of ISO data with respect to the previous results of Pezzuto et al. (1997) has improved the determination of some parameters such as the distance and the spectral type which are now in agreement with the values given by Drew et al. (1997). We also note that interferometric observations (Millan-Gabet et al. 2001) at near IR wavelengths ( $H$ and $K^{\prime}$ bands) are also consistent with a spherically dusty envelope around the central star even if a model with a flat blackbody face-on disc cannot be ruled out.

\section{2. $\mathrm{HI}$ recombination lines}

In the ISO-SWS spectrum $23 \mathrm{H}$ I recombination lines of the Brackett, Pfund and Humphreys series have been detected. Moreover observations of Brackett and Paschen lines are available in literature, in particular Thompson et al. (1977) measured the Brackett lines from Br10 to $\mathrm{Br} 20$ and $\mathrm{Br} \gamma$ plus the $\mathrm{Pa} \beta$ and $\mathrm{Pa} \gamma$ lines while McGregor et al. (1984) measured the Paschen lines from $\mathrm{Pa} \delta$ to Pa16. These H I recombination lines have been compared with a wind model (see Benedettini et al. 1998) which considers a spherically symmetric and fully ionized envelope with a constant rate of mass loss. The gas is assumed to be in LTE condition with a temperature of $T=10^{4} \mathrm{~K}$. The adopted gas velocity law is:

$v(r)=v_{\mathrm{i}}+\left(v_{\max }-v_{\mathrm{i}}\right)\left[1-\left(r_{*} / r\right)\right]$

where $v_{\mathrm{i}}=20 \mathrm{~km} \mathrm{~s}^{-1}$ is the initial velocity, $v_{\max }=$ $380 \mathrm{~km} \mathrm{~s}^{-1}$ is the maximum wind velocity (derived from the $\mathrm{H} \alpha$ observed profiles, Finkenzeller et al. 1984) and $r_{*}=3.2 \times 10^{11} \mathrm{~cm}$ is the stellar radius of a B1.5 ZAMS star (Thompson 1984). The envelope is assumed to be fully ionized only up to a distance $R$ from the central star (ionization bounded flow) and the star is positioned at a distance $D=250 \mathrm{pc}$. The observed line fluxes have been de-reddened by using the Rieke \& Lebofsky (1985) extinction law. The HI recombination lines in the SWS spectral range, essentially the Pfund and Humphreys lines at wavelengths greater than $2.3 \mu \mathrm{m}$, are not very sensitive to the changing in the extinction value, while the Brackett and Paschen lines, lying at shorter wavelength, are much more affected by the extinction. Therefore these latter lines are a good tool to constrain the visual extinction towards the star while the ISO-SWS lines have been used to constrain the mass loss rate and the size of the ionized region. Figure 4 shows the line ratios of the Paschen, Brackett, Pfund and Humphreys series with respect to the $\operatorname{Br} \alpha$ and the best fit model whose parameters are: mass loss rate $\dot{M}=9 \times 10^{-7} M_{\odot} \mathrm{yr}^{-1}$, radius of the ionized region $R=30 r_{*}$ and visual extinction $A_{\mathrm{V}}=7.5 \mathrm{mag}$. This latter is slightly smaller than the value of $8.3 \mathrm{mag}$ derived by Hillenbrand et al. (1992) and confirms the estimate obtained with the continuum data. A visual extinction less the 7 mag has been also suggested in literature, e.g.
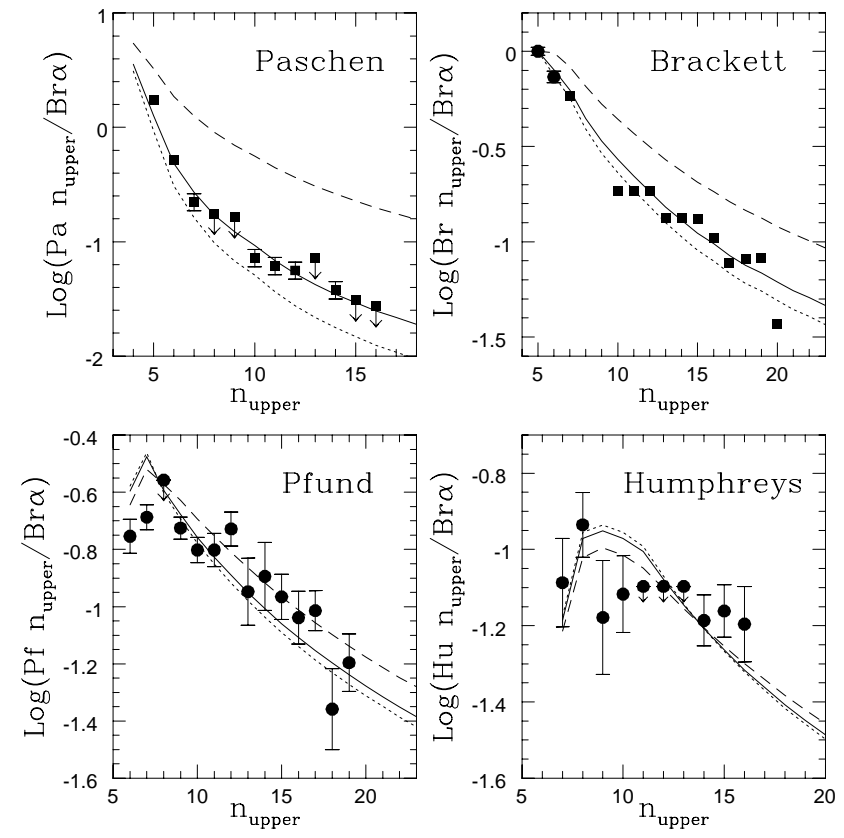

Fig. 4. Line ratios of the Paschen, Brackett, Pfund and Humphreys series with respect to the $\operatorname{Br} \alpha$ line; circles are the ISO-SWS observations while squares are ground based observations. Lines represent the model line ratios corresponding to $\dot{M}=9 \times 10^{-7} M_{\odot} \mathrm{yr}^{-1}$ and $R=30 r_{*}$; the model fluxes have been reddened with a visual extinction of 3 mag (dashed line), $7.5 \mathrm{mag}$ (solid line) and 9 mag (dotted line).

$A_{\mathrm{V}}=2.9$ mag by Cantó et al. (1984), but it must be excluded because it is not in agreement with the observed values of the Paschen and Brackett lines (see Fig. 4).

Also in this object as well as towards other HAEBE stars observed with ISO-SWS (i.e. MWC1080 and CoD- $42^{\circ} 11721$ Benedettini et al. $1998 ; \mathrm{BD}+40^{\circ} 4124$ van den Ancker et al. 2000), the line decrement of the H I recombination spectral series can be reproduced by assuming a ionization bounded compact region instead of a density bounded wind model.

From the absolute flux of the $\operatorname{Br} \alpha, \operatorname{Br} \gamma, \operatorname{Pf} \beta, \operatorname{Pf} \gamma$ and under the assumption of a distance of $450 \mathrm{pc}$ and of a density bounded flow, Nisini et al. (1995) found $\dot{M}=(1.25 \pm 0.05) \times 10^{-6} M_{\odot} \mathrm{yr}^{-1}$, slightly higher than the present result. If in our model we assume the distance of $450 \mathrm{pc}$ the corresponding best fit parameters are: $\dot{M}=2 \times 10^{-6} M_{\odot} \mathrm{yr}^{-1}, R=60 r_{*}$ and $A_{\mathrm{V}}=7.5 \mathrm{mag}$; the mass loss rate and the size of the emitting region differ by a of factor 2 from the values derived with $D=250 \mathrm{pc}$ while the visual extinction does not change.

The ionized region in which the $\mathrm{H}$ I recombination lines are produced emits also a free-free continuum in the radio range. Under the physical assumptions of our model, the radio continuum is related to the $\operatorname{Br} \alpha$ flux by the simple relation $S_{3.6 \mathrm{~cm}}(\mathrm{mJy})=$ $1.86 \times 10^{11}\left(100 / v_{\max }\left(\mathrm{km} \mathrm{s}^{-1}\right)\right)^{1 / 3} F_{\operatorname{Br} \alpha}\left(\mathrm{erg} \mathrm{cm}^{-2} \mathrm{~s}^{-1}\right)$ (Simon et al. 1983). By using the observed $\operatorname{Br} \alpha$ flux we derived $S_{3.6 \mathrm{~cm}}=8.9 \mathrm{mJy}$ consistent with the value measured by Skinner et al. (1993) (10 \pm 3 mJy from the 
average of three measurements). We conclude that the region responsible for the $\mathrm{H}$ I recombination lines is also responsible for the observed radio continuum emission.

\subsection{Silicate features}

The ground based photometry by Berrilli et al. (1992) has already revealed the presence of an absorption feature at $9.7 \mu \mathrm{m}$ in MWC 297 (see Fig. 1), but the authors attributed the observed decrement of the continuum level to a single absorption band while the ISO-SWS spectrum reveals the presence of two bands. Berrilli et al. (1992) estimated the optical depth of the 9.7 silicate absorption, $\tau_{9.7}=0.4$, from the N2 and N3 photometric bands supposing only one absorption band. A more accurate value of the peak optical depth can be derived from the ISO-SWS spectrum; however it is difficult to give a very precise estimate because of the difficulty in the determination of the real continuum level, especially at wavelengths longer than $10 \mu \mathrm{m}$. A first order estimate can be given by linearly fitting the local continuum: in this case the $9.7 \mu \mathrm{m}$ silicate optical depth is $\tau_{9.7}=0.29 \pm 0.08$.

The visual extinction can be derived by using the Rieke \& Lebofsky (1985) relation $A_{\mathrm{V}} / \tau_{9.7}=16.6 \pm 2.1$. From our $\tau_{9.7}$ estimate we obtain $A_{\mathrm{V}}=5 \pm 2 \mathrm{mag}$, which can be considered a lower limit to the real value since the presence of the broad adjacent $16.4 \mu \mathrm{m}$ absorption band probably leads to an underestimate of the continuum level which turns into an underestimate of the visual extinction. Taking into account these considerations, we can say that the extinction is compatible with the value derived by using both the $\mathrm{H}$ I recombination lines and the continuum data.

The position at $9.7 \mu \mathrm{m}$ of the absorption peak indicates that the silicate responsible for this absorption is mainly amorphous olivine. The other silicate band peaks at a wavelength $(16.4 \mu \mathrm{m})$ shorter than expected $(\sim 18 \mu \mathrm{m})$, and has an intensity higher than the $9.7 \mu \mathrm{m}$ absorption $(9.7 \mu \mathrm{m} / 16.4 \mu \mathrm{m}$ integrated intensity ratio is $\sim 0.4$ ), while theoretical studies (e.g. Draine \& Hyung 1984) predict that the absorption band at $\sim 10 \mu \mathrm{m}$ is stronger than that at $\sim 18 \mu \mathrm{m}$. However, in a review work by Nuth III \& Hecht (1990) it is shown that both the peak positions and the intensity ratio between the $10 \mu \mathrm{m}$ and the $18 \mu \mathrm{m}$ bands can assume very different values. In general, as silicate grains are annealed the peak of the $10 \mu \mathrm{m}$ band shifts at longer wavelengths while the peak of the $18 \mu \mathrm{m}$ band shifts at shorter wavelength and its absolute intensity increases. Several laboratory experiments indicate that the more the dust grains are processed the stronger the $18 \mu \mathrm{m}$ band is compared to the $10 \mu \mathrm{m}$ band. In particular, studies (Nuth III \& Moore 1988) of the silicate residual from proton irradiation of $\mathrm{Fe}(\mathrm{CO})_{5}-\mathrm{SiH}_{4}{ }^{-}$ $\mathrm{H}_{2} \mathrm{O}$ ices have shown an integrated absorption ratio of $10 \mu \mathrm{m} / 18 \mu \mathrm{m}$ less than 1 . Observational evidences supporting this thesis are given by the work of Demyk et al. (1999) which, analyzing a set of ISO-SWS data, found that the $10 \mu \mathrm{m} / 18 \mu \mathrm{m}$ ratio decreases from evolved stars to protostars being a tracer of the silicate processing. The very low ratio observed along the line of sight of MWC 297 and the shifted position at lower wavelength of the peak of the $18 \mu \mathrm{m}$ band can be an indication that we are observing "old" silicates even if in this case a shift at longer wavelengths would be expected for the $9.7 \mu \mathrm{m}$ feature.

\section{4. $\mathrm{H}_{2} \mathrm{O}$ and $\mathrm{CO}_{2}$ ices}

The detection of $\mathrm{H}_{2} \mathrm{O}$ and $\mathrm{CO}_{2}$ ices at $2.96 \mu \mathrm{m}$ and $4.27 \mu \mathrm{m}$ respectively, indicates the presence of dense molecular material along the line of sight of MWC 297. The $\mathrm{CO}_{2}$ and $\mathrm{H}_{2} \mathrm{O}$ peak optical depths are: $\tau_{\mathrm{CO}_{2}}=$ $0.11 \pm 0.03$ and $\tau_{\mathrm{H}_{2} \mathrm{O}}=0.04 \pm 0.02$. From those values we calculate the column densities of the two molecules by using the approximate formula:

$N=\frac{\tau \Delta \nu}{A}$

where $\Delta \nu$ is the $F W H M$ measured in $\mathrm{cm}^{-1}$ and $A$ is the integrated absorption cross section for the molecule, which is $A=7.6 \times 10^{-17} \mathrm{~cm} \mathrm{~mol}^{-1}$ for $\mathrm{CO}_{2}$ (Gerakines et al. 1995) and $A=2 \times 10^{-16} \mathrm{~cm} \mathrm{~mol}^{-1}$ for $\mathrm{H}_{2} \mathrm{O}$ (d'Hendecourt $\&$ Allamandola 1986). The derived column densities are: $N\left(\mathrm{CO}_{2}\right)=(4 \pm 1) \times 10^{16} \mathrm{~cm}^{-2}$ and $N\left(\mathrm{H}_{2} \mathrm{O}\right)=(2 \pm$ 1) $\times 10^{16} \mathrm{~cm}^{-2}$. The ratio $N\left(\mathrm{CO}_{2}\right) / N\left(\mathrm{H}_{2} \mathrm{O}\right)=2.0 \pm 1.5$ appears higher than the values usually observed by ISOSWS. Indeed from ISO-SWS observations of a sample of sources in different environment and evolutionary status, Gerakines et al. (1999) found a strong correlation between the column density of $\mathrm{CO}_{2}$ ice relative to the $\mathrm{H}_{2} \mathrm{O}$ ice, where the ratio $N\left(\mathrm{CO}_{2}\right) / N\left(\mathrm{H}_{2} \mathrm{O}\right)$ falls in the range $0.10-0.23$. The overabundance of the $\mathrm{CO}_{2}$ ice compared with the water ice would indicate a prevalence of the oxidation process on carbonaceous grains during the ices formation or a temperature difference between the two components.

\section{Conclusion}

The ISO-SWS full grating spectrum of the Herbig Be star MWC 297 has been presented. The spectrum is dominated by a strong continuum; in addition, emission lines from the $\mathrm{H}$ I recombination series, PAH emission and absorption by solid $\mathrm{CO}_{2}, \mathrm{H}_{2} \mathrm{O}$, and silicates have been detected. Some of the observed features show peculiar characteristics. The ratio between the column density of solid $\mathrm{CO}_{2}$ and $\mathrm{H}_{2} \mathrm{O}$ $\left(N\left(\mathrm{CO}_{2}\right) / N\left(\mathrm{H}_{2} \mathrm{O}\right)=2.0 \pm 1.5\right)$ is higher than the values usually observed by ISO-SWS (0.10-0.23) in different lines of sight. The silicate absorption band associated to the $\mathrm{O}-\mathrm{Si}-\mathrm{O}$ bending mode, peaks at wavelength lower than expected $(16.4 \mu \mathrm{m}$ rather than $\sim 18 \mu \mathrm{m})$ and has a total intensity higher than that of the $9.7 \mu \mathrm{m}$ band: we suggest that the silicates along the line of sight of MWC 297 are composed by processed dust grains. 
We have shown that the overall SED of MWC 297 from optical to radio wavelengths is compatible with a spherical distribution of the circumstellar matter.

The $23 \mathrm{H}$ I recombination lines of the Brackett, Pfund and Humphreys series observed with ISO-SWS plus the Brackett and Paschen lines observed from the ground have been fitted with a ionized wind model; the line decrement of the spectral series have been reproduced by assuming a ionization bounded compact region. The same region is also responsible for the radio flux observed by Skinner et al. (1993).

From the analysis carried out on the ISO data, we derived a consistent set of physical parameters for the matter surrounding MWC 297 and for the object itself: $\mathrm{SpT} \simeq \mathrm{B} 2$, distance $D \simeq 280 \mathrm{pc}$, visual extinction $A_{\mathrm{V}}=7.5 \mathrm{mag}$, index of the temperature and density profile $q=0.5$ and $p=1.1$ respectively, mass loss rate $\dot{M}=9 \times 10^{-7} M_{\odot} \mathrm{yr}^{-1}$, radius of the ionized circumstellar region $R=30 r_{*}$ and optical depth at $9.7 \mu \mathrm{m}$ $\tau_{9.7}=0.29 \pm 0.08$.

Acknowledgements. We acknowledge Prof. F. Strafella for his contribution to the analysis of the continuum data and Prof. G. Strazzulla for the useful discussion about the ice features.

\section{References}

Andrillat, Y., \& Jaschek, C. 1998, A\&ASS, 131, 479

Benedettini, M., Nisini, B., Giannini, T., et al. 1998, A\&A, 339, 159

Bergen, Y. K., Kozlov, V. P., Krivstov, A. A., et al. 1988, Astrophys., 28, 529

Berrilli, F., Corciulo, G., Ingrosso, G., et al. 1992, ApJ, 398, 254

Cantó, J., Rodríguez, L. F., Calvet, N., \& Levreault, R. M. 1984, ApJ, 282, 631

Clegg, P. E., Ade, P. A. R., Armand, C., et al. 1996, A\&A, 315, L38

d'Hendecourt, L. B., \& Allamandola, L. J. 1986, A\&AS, 64, 453

de Graauw, T., Haser, L. N., Beintema, D. A., et al. 1996, A\&A, 315, L49

Demyk, K., Jones, A. P., Dartois, E., Cox, P., \& D'Hendecourt, L. 1999, A\&A, 349, 267
Di Francesco, J., Evans II, N. J., Harvey, P. M., Mundy, L. G., \& Butner, H. M. 1998, ApJ, 509, 324

Draine, B. T., \& Hyung, M. L. 1984, ApJ, 285, 89

Drew, J. E., Busfield, G., Hoare, M. G., et al. 1997, MNRAS, 286,538

Finkenzeller, U., \& Mundt, R. 1984, A\&AS, 55, 109

Gerakines, P. A., Schutte, W. A., Greenberg, J. M., \& van Dishoeck, E. F. 1995, A\&A, 296, 810

Gerakines, P. A., Whittet, D. C. B., Ehrenfreund, P., et al. 1999, ApJ, 522, 357

Henning, Th., Burkert, A., Launhardt, R., Leinert, C., \& Stecklum, B. 1998, A\&A, 336, 565

Herbig, G. H. 1960, ApJS, 4, 337

Hillenbrand, L. A., Strom, S. E., Vrba, F. J., \& Keene, J. 1992, ApJ, 397, 613

Hollenbach, D., \& McKee, C. F. 1989, ApJ, 342, 306

Kaufman, M. J., Wolfire, M. G., Hollenbach, D. J., \& Luhman, M. 1999, ApJ, 527, 795

Kessler, M. F., Steinz, J. A., Anderegg, M. E., et al. 1996, A\&A, 315, L27

Leech, K., et al. 1997, SWS Instrument Data User Manual, Issue 3.1, SAI/95-221/Dc

Lorenzetti, D., Tommasi, E., Giannini, T., et al. 1999, A\&A, 346,604

McGregor, P. J., Persson, S. E., \& Cohen, J. G. 1984, ApJ, 286,609

Meeus, G., Waters, L. B. F. M., Bouwman, J., et al. 2001, A\&A, 365, 476

Millan-Gabet, R., Schloerb, F. P., \& Traub, W. A. 2001, ApJ, 546,358

Nisini, B., Milillo, A., Saraceno, P., \& Vitali, F. 1995, A\&A, 302,169

Nuth III, J. A., \& Moore, M. H. 1988, ApJ, 329, L113

Nuth III, J. A., \& Hecht, J. H. 1990, Ap\&SS, 163, 79

Oudmaijer, R. D., \& Drew, J. E. 1999, MNRAS, 305, 166

Pezzuto, S., Strafella, F., \& Lorenzetti, D. 1997, ApJ, 485, 290

Rieke, G. H., \& Lebofsky, M. J. 1985, ApJ, 288, 618

Simon, M., Felli, M., Cassar, L., et al. 1983, ApJ, 266, 623

Skinner, S. L., Brown, A., \& Stewart, R. T. 1993, ApJS, 87, 217

Thompson, R. I., Strittmatter, P. A., Erickson, E. F., Witteborn, F., \& Strecker, D. W. 1977, ApJ, 218, 170

Thompson, R. I. 1984, ApJ, 283, 165

Valentijn, E. A., Feuchtgruber, H., Kester, D. J. M., et al. 1996, A\&A, 315, L60

van den Ancker, M. E., Wesselius, P. R., \& Tielens, A. G. G. M. 2000, A\&A, 355, 194 